\section{ORIGINAL RESEARCH}

\section{R. Zivadinov}

R. Galeotti

D. Hojnacki

E. Menegatti

M.G. Dwyer

C. Schirda

A.M Malagoni

K. Marr

C. Kennedy

I. Bartolomei

C. Magnano

F. Salvi

B. Weinstock-Guttman

P. Zamboni

\title{
Value of MR Venography for Detection of Internal Jugular Vein Anomalies in Multiple Sclerosis: A Pilot Longitudinal Study
}

BACKGROUND AND PURPOSE: CCSVI was recently described in patients with MS. CCSVI is diagnosed noninvasively by Doppler sonography and invasively by catheter venography. We assessed the role of conventional MRV for the detection of IJV anomalies in patients with MS diagnosed with CCSVI and in healthy controls who underwent MRV and Doppler sonography examinations during 6 months

MATERIALS AND METHODS: Ten patients with MS underwent TOF, TRICKS, Doppler sonography, and catheter venography at baseline. They were treated at baseline with percutaneous angioplasty and re-evaluated 6 months' posttreatment with MRV and Doppler sonography. In addition, 6 healthy controls underwent a baseline and a 6-month follow-up evaluation by Doppler sonography and MRV.

RESULTS: At baseline, the sensitivity, specificity, PPV, and NPV of Doppler sonography for detecting IJV abnormalities relative to catheter venography in patients with MS were calculated, respectively, at $82 \%$, $100 \%, 99 \%$, and $95 \%$. The figures were $99 \%, 33 \%, 33 \%, 99 \%$ for TOF and $99 \%, 39 \%, 35 \%$, and $99 \%$ for TRICKS. Venous anomalies included the annulus, septum, membrane, and malformed valve. No agreement was found between TOF and catheter venography in $70 \%$ of patients with MS and between TRICKS and catheter venography in $60 \%$ of patients with MS. At follow-up, $50 \%$ of the patients with MS presented with abnormalities on Doppler sonography but only $30 \%$ were diagnosed with restenosis.

CONCLUSIONS: Conventional MRV has limited value for assessing IJV anomalies for both diagnostic and posttreatment purposes.

ABBREVIATIONS: $\mathrm{CCSVI}=$ chronic cerebrospinal venous insufficiency; $\mathrm{CV}=$ catheter venography: EVTMS = Endovascular Treatment Study for MS; DS = Doppler sonography; IJV = internal jugular vein; LIJV = left internal jugular vein; 6 mo $=6$-month follow-up examination; MRV $=$ MR venography; $\mathrm{MS}=$ multiple sclerosis; NPV = negative predictive value; PPV = positive predictive value; PTA = percutaneous transluminal angioplasty; RIJV = right internal jugular vein; $T O F=2 D$ time-of-flight venography; TRICKS $=3 \mathrm{D}$ time-resolved imaging of contrast kinetics

C CSVI was recently described in patients with MS. ${ }^{1}$ This vascular condition is characterized by multiple intraluminal stenosing malformations of the principal pathways of extracranial venous drainage, particularly the IJVs and the azygous vein, which restrict normal outflow of blood from the brain. ${ }^{1-4}$ This condition can be diagnosed noninvasively by using Doppler sonography ${ }^{1,2,5-7}$ and invasively by using catheter venography. ${ }^{1,3,6}$

In previous studies, by using Doppler sonography and catheter venography, ${ }^{1,3}$ multiple anomalies of the veins outside the skull and spine have been described. These irregularities include the annulus, septum/valve malformations, hypoplasia, twisting, and membranous obstruction or agenesis of a venous segment. The transcranial and extracranial echo color Doppler sonography protocol, which allows noninvasive measurement of venous he-

Received July 26, 2010; accepted after revision September 8.

From the Buffalo Neuroimaging Analysis Center (R.Z., M.G.D., C.S., K.M., C.K., C.M.), State University of New York, Buffalo, New York; Jacobs Neurological Institute (R.Z., D.H., B.W.-G.), State University of New York, Buffalo, New York; and Vascular Diseases Center (R.G., E.M., A.M.M., I.B., F.S., P.Z.), University of Ferrara-Bellaria Neurosciences, Ferrara and Bologna, Italy.

This study was supported in part by the Hilarescere Foundation and the Buffalo Neuroimaging Analysis Center.

Please address correspondence to Robert Zivadinov, MD, PhD, Department of Neurology, School of Medicine and Biomedical Sciences, Buffalo Neuroimaging Analysis Center, 100 High St, Buffalo, NY 14203; e-mail: rzivadinov@bnac.net

Indicates open access to non-subscribers at www.ajnr.org

DOI 10.3174/ajnr.A2386 modynamic parameters indicative of CCSVI, was developed ${ }^{1}$ and validated in preliminary studies. ${ }^{2,6}$

MRV could be another noninvasive diagnostic tool for studying the venous system of the head and neck. ${ }^{4}$ However, at the moment, there is a lack of experience and standards in the use of this technique for the detection of cerebrospinal venous anomalies and there are no available follow-up studies with MRV in patients with MS.

PTA was proposed for treatment of CCSVI. ${ }^{3}$ In a recent study, vein patency was assessed by using a diagnostic Doppler sonography protocol at $1,3,6,12,15$, and 18 months after treatment. ${ }^{3}$ If restenosis was suspected after 18 months, a second catheter venography was performed, as well as a second PTA treatment, if needed. Researchers found an elevated rate of restenosis in the IJVs at 18 months post-PTA (47\%) compared with the azygous vein.

The present longitudinal study describes the findings of Doppler sonography, catheter venography, and MRV pre-PTA treatment in patients with MS and their 6-month follow-up with Doppler sonography and MRV post-PTA. These results are compared with a group of healthy controls who were also followed for 6 months both with Doppler sonography and MRV.

\section{Materials and Methods}

\section{Subjects}

A subgroup of 10 patients with MS who participated in the EVTMS 8 underwent an MRV examination at baseline, in addition to Doppler 
sonography and catheter venography. They were diagnosed with CCSVI (as evidenced by Doppler sonography and catheter venography) and treated with PTA when significant abnormalities were detected. They were re-evaluated 6 months' posttreatment with MRV and Doppler sonography. The inclusion criteria were the following: CCSVI as defined by presence of $\geq 2$ venous hemodynamic criteria ${ }^{1}$; diagnosis of relapsing-remitting $\mathrm{MS}^{9}$; Expanded Disability Status Scale score ${ }^{10}$ range between 0 and 5.5; age of $18-65$ years; disease duration between 5 and 10 years; current treatment with US Food and Drug Administration-approved disease-modifying therapies; normal renal function (creatinine clearance of $>58 \mathrm{~mL} / \mathrm{min}$ ); and no medical contraindications for PTA. Exclusion criteria were acute relapse and/or steroid treatment within the 30 days preceding study entry, pre-existing medical conditions associated with brain pathology (eg, neurodegenerative disorder, positive history of alcohol abuse, and so forth), and history of cerebral congenital vascular malformations (Klippel-Trenaunay, Parkes-Weber, Servelle-Martorell, and Budd-Chiari syndromes). In addition, 6 healthy controls underwent a baseline and a 6-month follow-up evaluation by Doppler sonography and MRV.

The study was approved by the local institutional review board, and informed consent was obtained from all study subjects.

\section{MRV}

All subjects were examined on a 3T Signa Excite HD 12.0 TwinSpeed 8-channel scanner (GE Healthcare, Milwaukee, Wisconsin), with a maximum slew rate of $150 \mathrm{~T} / \mathrm{m} / \mathrm{s}$ and a maximum gradient amplitude in each orthogonal plane of $50 \mathrm{mT} / \mathrm{m} / \mathrm{ms}$ (zoom mode). A multichannel head and neck coil manufactured by GE Healthcare was used to acquire the following sequences: TOF venography and TRICKS MRV. The parameters used for TOF were the following: TR/TE, 17/4.3 ms; flip angle, $70^{\circ}$; 1.5 -mm section thickness; matrix, 320/192; and acquisition in the axial scanning plane. The parameters used for TRICKS were the following: TR/TE, $4.2 / 1.6 \mathrm{~ms}$; flip angle, $30^{\circ}$; $2-\mathrm{mm}$ section thickness; matrix, 320/192; and acquisition in the coronal scanning plane. Intravenous gadolinium contrast was injected at a rate of 2 $\mathrm{mL} / \mathrm{s}$ by using a pressure injector. The total volume of contrast was 20 $\mathrm{mL}$. The scanning protocol consisted of 18 phases of acquisition, each of 5 seconds' duration.

The flow morphology of the IJVs was assessed on axial source images on TOF as well as on axial reconstructed TRICKS sections. The flow was evaluated on an ordinal scale ranging from absent (no visible flow) to ellipsoidal (patent lumen). We assigned 5 qualitative flow categories: absent, pinpoint, flattened, crescentic, and ellipsoidal. Because the morphology of the IJV can vary along the vessel, we considered the narrowest point in both the inferior and the superior segments, respectively. Absent and pinpoint IJV flows were considered abnormal.

All MR imaging scans were examined by 2 independent neuroradiologists in a blinded manner.

The IJV MRV variable used for comparison with Doppler sonography and catheter venography was abnormal/normal flow. We considered comparing the asymmetries and prominence of the other most important veins in the neck visible on MRV with Doppler sonography and catheter venography, but found it difficult to assess the differences without a specific predefined Doppler sonography and catheter venography assessment protocol, which was not included in this study.

\section{Doppler Sonography}

Cerebral venous return was examined by an expert technologist at baseline and at 6 months by using an echo color Doppler sonography scanner (ECD Esaote-Biosound Mylab 25; Esaote, Genoa, Italy) equipped with 2.5- and 7.5- to $10-\mathrm{MHz}$ transducers, with the subject positioned on a bed tilted at $90^{\circ}$ and $0^{\circ}$. All subjects were scanned following the established protocol for a diagnosis of CCSVI, ${ }^{1,2}$ consisting of transcranial and extracranial color echo Doppler sonography to measure the following 5 venous hemodynamic parameters indicative of CCSVI: 1) reflux in the IJVs and/or in the VVs in sitting and in supine positions ( 90 and $0^{\circ}$ ) ("reflux" is defined as flow directed toward the brain for a duration of $>0.88$ seconds); 2 ) reflux in the deep cerebral vein ("reflux" is defined as reverse flow for a duration of 0.5 seconds in 1 of the DCVs: the internal cerebral vein, the basal vein of Rosenthal, or the vein of Galen); 3) B-mode abnormalities or stenoses in the IJVs ("IJV stenosis" is defined as a cross-sectional area of this vein $\leq 0.3 \mathrm{~cm}^{2}$; flaps, webs, septa, and so forth in the lumen of IJVs are considered B-mode abnormalities that significantly disturb cerebral venous outflow); 4) flow that is not Doppler-detectable in the IJVs and/or vertebral veins despite multiple deep breaths; and 5) reverted postural control of the main cerebral venous outflow pathway by measuring the difference in the cross-sectional area of the IJVs between the supine and upright positions. A subject was considered CCSVI-positive, if $\geq 2$ venous hemodynamic criteria were fulfilled, as previously proposed. ${ }^{1}$

When comparing Doppler sonography findings with MRV and catheter venography findings, the presence of at least 1 of the following IJV venous hemodynamic anomalies was considered an abnormal examination finding: B-mode abnormalities (flaps, membrane, and malformed valve, septa, web), stenoses, absence of detectable flow, and the presence of reflux in both sitting and supine positions.

The Doppler sonography examination was also used to determine whether the patients presented with significant restenosis after the PTA. The restenosis was defined as the presence of $\geq 2$ venous hemodynamic criteria, as previously proposed. ${ }^{1}$

\section{Catheter Venography and PTA}

Catheter venography was performed only in patients with MS after the Doppler sonography examination showed that all patients with MS fulfilled $\geq 2$ venous hemodynamic criteria. ${ }^{1}$ It was performed via catheterization of the left iliac femoral vein and comprised visualization of the lumbar veins, left renal vein, azygous vein, and IJVs. ${ }^{3}$ Significant stenosis was considered to be any venous lumen reduction $>50 \%$. We investigated the following IJV anomalies: annulus, a significant circumferential stenosis of the venous wall; septum/valve malformation, anomalous valve apparatus causing significant flow obstacles at the level of the junction of the brachiocephalic trunk; membranous obstruction, a membrane almost completely occluding a vein; hypoplasia, an underdeveloped long venous segment; twisting, severe stenosis as a consequence of a twisted venous segment; and agenesis, complete anatomic absence of a venous segment.

Catheter venography was conducted by an interventional radiologist. The presence of at least 1 of these anomalies in the IJVs was considered an abnormal examination finding when compared with MRV and Doppler sonography. Catheter venography was used as a criterion standard for comparison with MRV and Doppler sonography.

PTA was performed exclusively at the levels of the azygous vein and IJVs when significant stenoses were detected; compliant-type balloon catheters were used. At the end of the therapeutic procedure, a 


\begin{tabular}{|c|c|c|c|c|c|c|c|c|}
\hline \multirow{2}{*}{$\begin{array}{l}\text { Patients } \\
\text { with MS }\end{array}$} & \multicolumn{2}{|c|}{ 2D-TOF } & \multicolumn{2}{|c|}{ 3D-TRICKS } & \multicolumn{2}{|c|}{ DS } & \multicolumn{2}{|c|}{$\mathrm{CV}$} \\
\hline & RIJV & LIJV & RIJV & LIJV & RIJV & LIJV & RIJV & LIJV \\
\hline 1-baseline & Normal & Normal & Normal & Abnormal & Abnormal & Abnormal & Normal & Abnormal \\
\hline $1-6$ mo & Normal & Normal & Normal & Normal & Normal & Normal & - & - \\
\hline 2-baseline & Normal & Normal & Abnormal & Abnormal & Abnormal & Abnormal & Abnormal & Abnormal \\
\hline 2-6 mo & Abnormal & Abnormal & Abnormal & Abnormal & Abnormal & Abnormal & - & - \\
\hline 3-baseline & Normal & Normal & Normal & Normal & Abnormal & Abnormal & Abnormal & Abnormal \\
\hline 3- 6 mo & Normal & Normal & Normal & Normal & Normal & Abnormal & - & - \\
\hline 4-baseline & Normal & Normal & Normal & Normal & Normal & Abnormal & Normal & Abnormal \\
\hline 4-6 mo & Normal & Normal & Abnormal & Abnormal & Abnormal & Normal & - & - \\
\hline 5-baseline & Normal & Normal & Normal & Normal & Abnormal & Normal & Abnormal & Normal \\
\hline 5-6 mo & Normal & Normal & Normal & Normal & Abnormal & Normal & - & - \\
\hline 6-baseline & Normal & Normal & Normal & Normal & Abnormal & Abnormal & Abnormal & Abnormal \\
\hline 6-6 mo & Normal & Normal & Normal & Normal & Abnormal & Abnormal & - & - \\
\hline 7-baseline & Normal & Abnormal & Normal & Abnormal & Normal & Abnormal & Normal & Abnormal \\
\hline $7-6$ mo & Normal & Abnormal & Abnormal & Abnormal & Normal & Normal & - & - \\
\hline 8-baseline & Abnormal & Abnormal & Abnormal & Normal & Abnormal & Abnormal & Abnormal & Abnormal \\
\hline 8-6 mo & Abnormal & Abnormal & Abnormal & Abnormal & Abnormal & Abnormal & - & - \\
\hline 9-baseline & Normal & Abnormal & Normal & Normal & Abnormal & Abnormal & Abnormal & Abnormal \\
\hline 9-6 mo & Abnormal & Abnormal & Normal & Abnormal & Abnormal & Abnormal & - & - \\
\hline 10-baseline & Normal & Normal & Normal & Normal & Abnormal & Abnormal & Abnormal & Abnormal \\
\hline 10- 6 mo & Normal & Normal & Normal & Normal & Normal & Normal & - & - \\
\hline
\end{tabular}

a Absent and pinpoint IJV flow was considered abnormal on TOF and TRICKS; the presence of at least 1 of the following IJV parameters was considered abnormal on a DS examination: B-mode abnormalities (flaps, septa, web), stenoses, absence of detectable flow, and presence of reflux in both sitting and supine positions. On CV, the presence of stenosis $>50 \%$ of the IJV diameter or at least 1 of the following anomalies was considered abnormal: annulus, septum/valve malformation, hypoplasia, twisting, membrane, and agenesis.

catheter venography of the azygous vein and/or IJVs was repeated to document the immediate outcome of the treatment.

\section{Statistical Analysis}

Differences among groups were tested for significance by using $\chi^{2}$ tests, the 1 -sided Fisher exact test, and a Student $t$ test. Cohen $\kappa$ was used for calculation of the agreement between different imaging techniques of normal or abnormal IJV findings in healthy controls and patients with MS. By considering the catheter venography findings in patients with MS as a criterion standard, we calculated — for the left and right IJVs — the sensitivity, specificity, accuracy, PPV, and NPV of TRICKS, TOF, and Doppler sonography. Thus, a "true-positive" was defined as an abnormal imaging finding on TRICKS, TOF, or Doppler sonography and the presence of an abnormal IJV on catheter venography; a "false-positive" was defined as an abnormal imaging finding on those techniques in the absence of an abnormality on catheter venography. A "falsenegative" was defined as a normal imaging finding and the presence of an abnormality on catheter venography, and a "true-negative" was defined as a normal imaging finding in the absence of an abnormality on catheter venography.

Data were considered significant at the $P<.05$ level by using 2-tailed tests. No corrections for multiple comparisons were made, given the small sample size and pilot nature of the study.

\section{Results}

\section{Demographic and Clinical Characteristics}

The mean age of the patients with MS was $35.4 \pm 7.2$ years; mean disease duration, $7.5 \pm 1.8$ years; and median Expanded Disability Status Scale score, 2.5. Sixty percent of the patients with MS were women. The mean age of the HC group was $34.5 \pm 7.6$ years, and the proportion of women was $66 \%$. All patients with MS were on disease-modifying therapy (4 were on subcutaneous interferon $\beta$-1a, 2 were on intramuscular interferon $\beta$-1a, 2 were on natalizumab, and 2 were on glatiramer acetate).

\section{IJV Findings in Patients with MS and Healthy Controls at Baseline and Follow-Up}

Table 1 and Figs 1 and 2 show TOF, TRICKS, Doppler sonography, and catheter venography findings, at baseline and follow-up, in the left and right IJVs of patients with MS. At baseline, abnormalities were found in all patients with MS on Doppler sonography and catheter venography, in 30\% of patients on TOF, and in $40 \%$ on TRICKS. All patients presented with $\geq 2$ venous hemodynamic criteria on Doppler sonography, and the mean number of venous hemodynamic criteria was 4 . Catheter venography of the right IJV showed the presence of an annulus in 4 patients, a septum in 3 patients, and no abnormalities in 3 patients. Catheter venography findings of the patients with MS in the left IJV were the following: annulus (5), septum (3), membrane (1), malformed valve (1), and normal examination findings (1).

All patients with MS underwent PTA at baseline. At followup, $50 \%$ of the patients with MS presented with abnormalities on Doppler sonography but only $30 \%$ presented with $\geq 2$ venous hemodynamic criteria and were diagnosed with restenosis. Flow abnormalities were found in $40 \%$ of patients on TOF and $50 \%$ on TRICKS at the 6-month follow-up.

Table 2 and Figs 3 and 4 show TOF, TRICKS, and Doppler sonography findings in healthy controls at baseline and at 6-month follow-up. No healthy control presented with $\geq 2$ venous hemodynamic criteria on Doppler sonography, and the mean number of venous hemodynamic criteria was zero. At baseline, individual Doppler sonography abnormalities were found in 2 healthy controls, in 1 healthy control on TOF, and in 1 on TRICKS. At follow-up, 2 healthy controls showed 

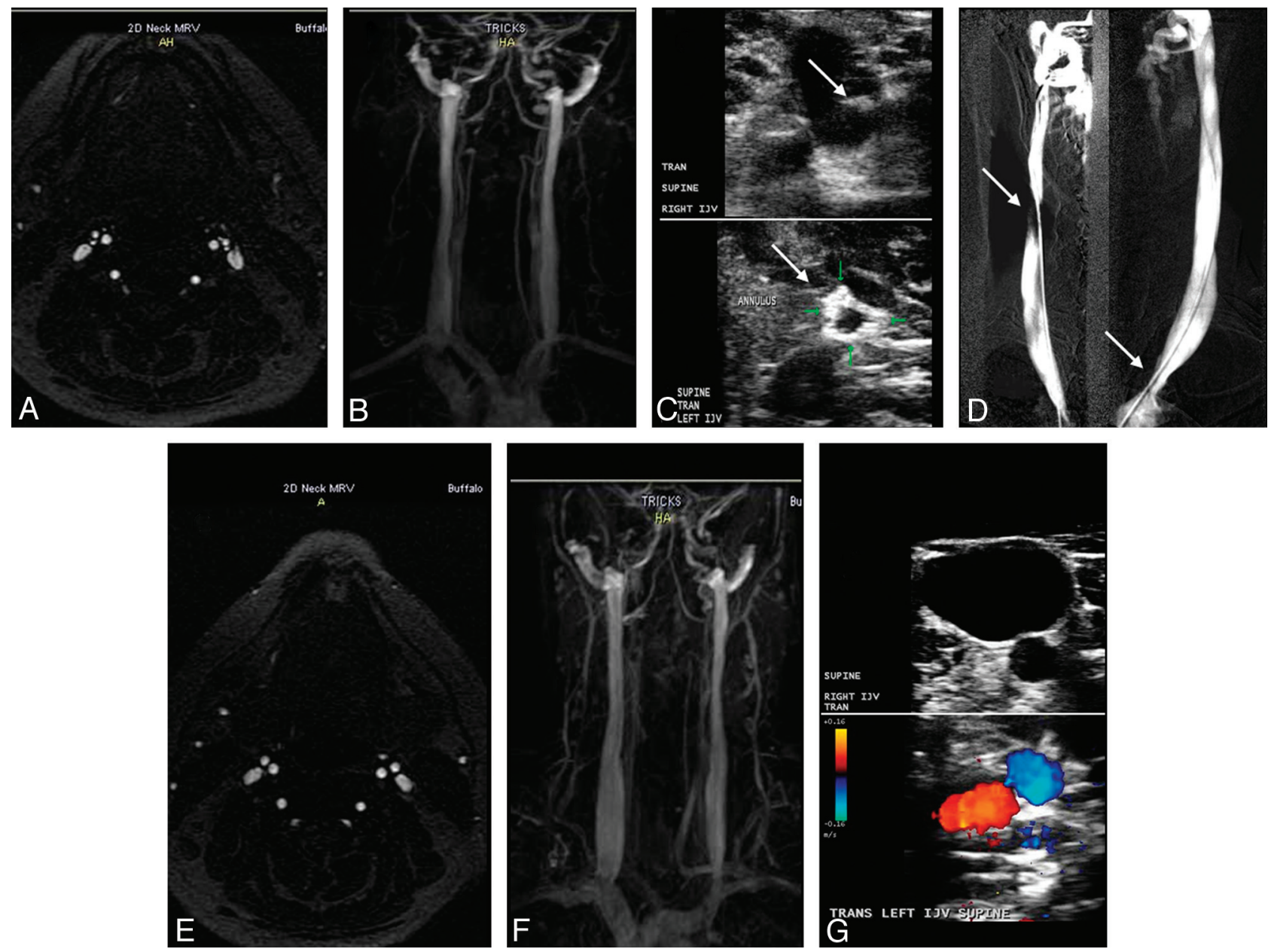

Fig 1. Patient with MS (a 43-year-old woman) shows normal examination findings on TOF $(A)$ and TRICKS $(B)$ of both IJVs pretreatment. $C$, Doppler sonography shows the presence of a septum (arrow) in the right IJV (upper image) and an annulus (arrow) in the left IJV (lower image). D-F, Catheter venography (D) confirms the presence of a septum (arrow) in the right IJV and an annulus (arrow) in the left IJV. The posttreatment 6-month follow-up shows normal examination findings on TOF (E), TRICKS (F), and Doppler sonography (G).

abnormalities on TOF: 1 on TRICKS and 1 on Doppler sonography.

\section{Sensitivity, Specificity, and Positive and Negative} Predictive Values between Different Imaging Modalities at Baseline Relative to Catheter Venography

The sensitivity, specificity, PPV, and NPV of Doppler sonography for detecting IJV abnormalities relative to catheter venography were $82 \%, 100 \%, 99 \%$, and $95 \%$ (Table 3 ), respectively. The figures were, respectively, 99\%, 33\%, 33\%, and 99\% for TOF and 99\%, 39\%, 35\%, and 99\% for TRICKS (Table 3$)$.

\section{Agreement between Different Imaging Techniques at Baseline and Follow-Up}

In patients with MS, the agreement between Doppler sonography and catheter venography findings was $100 \%$ in the left IJV $(\kappa=1)$ and $90 \%$ in the right IJV $(\kappa=0.737)$. There was no agreement between TOF and catheter venography findings in $70 \%$ of patients with MS and no agreement between TRICKS and catheter venography in $60 \%$ of patients with MS. Similar figures were observed when TOF and TRICKS were compared with Doppler sonography.

When both patients with MS and healthy controls were examined, there was no agreement between TOF and Doppler sonography findings in $56.2 \%$ of subjects and no agreement in $50 \%$ of subjects between TRICKS and Doppler sonography. No laterality differences were observed. There was strong agreement between MRV findings (TOF versus TRICKS) in $93.8 \%$ of the subjects $(\kappa=0.765)$ in the right IJV but not in the left IJV $(\kappa=0.179)$.

At follow-up, strong agreement was found in patients with MS in the left IJV between TOF and TRICKS $(\kappa=0.783)$ but not in the right IJV $(\kappa=0.286)$. No significant agreement was found at follow-up in patients with MS between either TOF or TRICKS and Doppler sonography findings.

In healthy controls, there was no significant agreement at follow-up between either TOF or TRICKS and Doppler sonography findings. However, complete agreement was detected between TOF and TRICKS in the left IJV $(\kappa=1)$, but not in the right IJV.

\section{Discussion}

This pilot study investigated the value of extracranial MRV for the assessment of IJV anomalies in patients with MS and healthy controls. In patients with MS and healthy controls, TRICKS and TOF showed no agreement with Doppler sonography findings of the IJVs both at baseline and follow-up. 

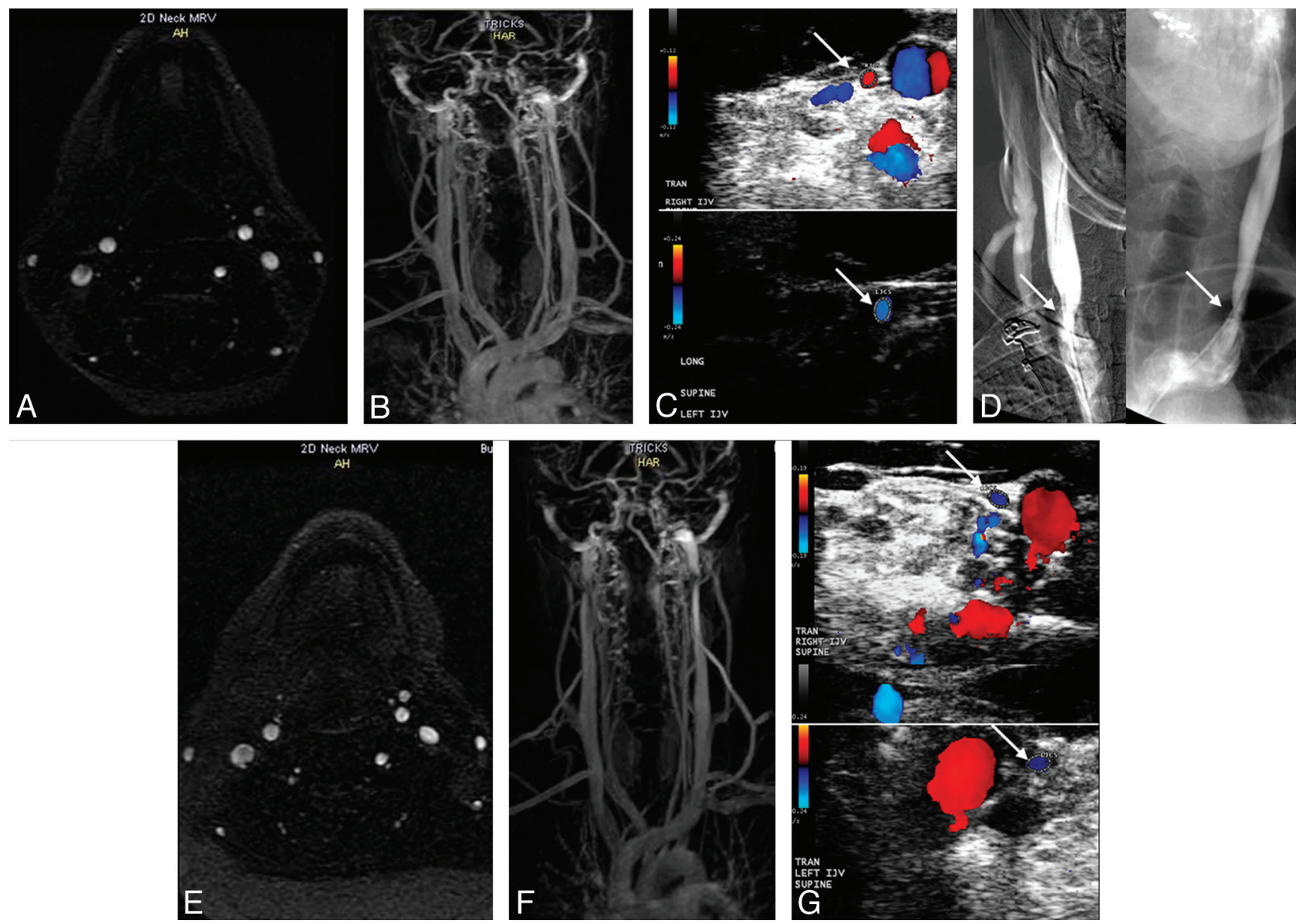

Fig 2. Patient with MS (a 44-year-old man) has normal examination findings on TOF $(A)$ and TRICKS $(B)$ of both IJVs pretreatment. $C$, Doppler sonography shows the presence of stenoses in the right IJV (arrow, upper image) and in the left IJV (arrow, lower image). D, Catheter venography confirms the presence of stenosis (arrows) in the right IJV and in the left IJV. E-G, The posttreatment 6-month follow-up shows normal examination findings on TOF ( $E$ ) and TRICKS ( $F$ ), but no change in the stenosis (arrows) on Doppler sonography (G) in the right IJV (upper image) and in the left IJV (lower image).

Table 2: MRV and Doppler sonography findings in healthy controls at baseline and 6-month follow-up ${ }^{\mathrm{a}}$

\begin{tabular}{|c|c|c|c|c|c|c|}
\hline \multirow{2}{*}{$\begin{array}{l}\text { Healthy } \\
\text { Controls }\end{array}$} & \multicolumn{2}{|c|}{ TOF } & \multicolumn{2}{|c|}{ TRICKS } & \multicolumn{2}{|c|}{ DS } \\
\hline & RIJV & LIJV & RIJV & LIJV & RIJV & LIJV \\
\hline 1-baseline & Normal & Normal & Normal & Normal & Normal & Normal \\
\hline $1-6$ mo & Normal & Normal & Normal & Normal & Normal & Normal \\
\hline 2-baseline & Normal & Normal & Normal & Normal & Normal & Normal \\
\hline 2-6 mo & Normal & Abnormal & Normal & Abnormal & Normal & Normal \\
\hline 3-baseline & Normal & Normal & Normal & Normal & Normal & Normal \\
\hline 3-6 mo & Normal & Normal & Normal & Normal & Normal & Normal \\
\hline 4-baseline & Normal & Normal & Normal & Normal & Normal & Normal \\
\hline $4-6$ mo & Normal & Normal & Normal & Normal & Normal & Normal \\
\hline 5-baseline & Abnormal & Normal & Abnormal & Normal & Normal & Abnormal \\
\hline 5-6 mo & Normal & Normal & Abnormal & Normal & Abnormal & Normal \\
\hline 6-baseline & Normal & Normal & Normal & Normal & Normal & Abnormal \\
\hline 6-6 mo & Normal & Normal & Normal & Normal & Normal & Abnormal \\
\hline
\end{tabular}

a Absent and pinpoint IJV flow was considered abnormal on TOF and TRICKS; the presence of at least 1 of the following IJV parameters was considered abnormal on a DS examination: B-mode abnormalities (flaps, septa, and web), stenoses, absence of detectable flow, and presence of reflux in both sitting and supine positions.

There was low specificity and PPV and no agreement between the $2 \mathrm{MRV}$ techniques and catheter venography findings in patients with MS at baseline. On the other hand, Doppler sonography showed high specificity and PPV, as well as strong agreement with catheter venography findings at baseline. These results indicate that conventional MRV has limited value for the detection of venous abnormalities cross-sectionally and longitudinally. Given the low accuracy of conven- tional MRV for depicting cross-sectional and longitudinal IJV anomalies, MRV findings should be interpreted with caution, both for diagnosis and monitoring treatment outcome.

A recently published longitudinal study in patients with MS diagnosed with CCSVI and treated with PTA used Doppler sonography and catheter venography for a diagnosis of CCSVI. ${ }^{3}$ The present study was based on a subcohort of patients with MS who participated in the EVTMS ${ }^{8}$ and 

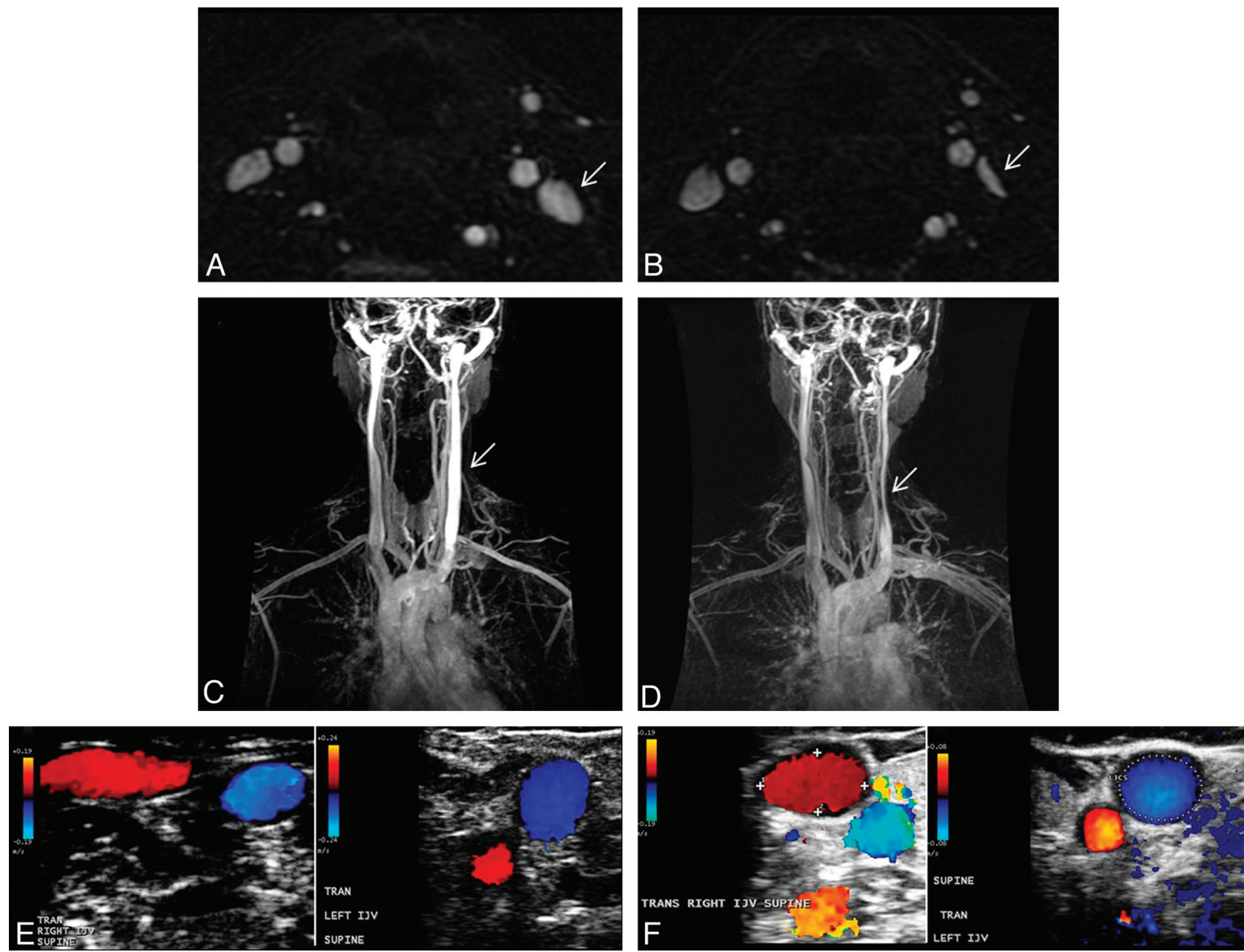

Fig 3. Variability between the baseline $(A, C$, and $E$, TOF, TRICKS, and Doppler sonography, respectively) and follow-up $(B, D$, and $F$, TOF, TRICKS, and Doppler sonography, respectively) examinations in a 42-year-old healthy female control. Flattening of the left IJV (arrows) at follow-up is noted on the TOF (B) and TRICKS (D), whereas Doppler sonography shows normal examination findings like those at baseline $(\boldsymbol{F})$.

underwent MRV at baseline, in addition to Doppler sonography and catheter venography. At 6-month follow-up, they were assessed with MRV and Doppler sonography. We did not perform a follow-up catheter venography in patients with MS to confirm the Doppler sonography 6-month findings. In addition, a group of 6 healthy controls was enrolled to increase confidence in the detection of IJV venous anomalies and to test the longitudinal stability of MRV and Doppler sonography.

At baseline, as per inclusion criteria, all patients with MS had to present $\geq 2$ venous hemodynamic criteria and meet a diagnosis of CCSVI to be enrolled in the EVTMS. ${ }^{1}$ Catheter venography confirmed the venous anomalies identified by Doppler sonography in the IJVs in $100 \%$ of patients with MS on the left and $90 \%$ on the right, resulting in a strong agreement between the 2 imaging techniques $(\kappa=0.737-1)$. The sensitivity, specificity, PPV, and NPV were also very high (Table 3). The venous anomalies consisted of the annulus, septum, membrane, and malformed valve. PTA was performed exclusively at the levels of the azygous vein and IJVs when these anomalies were detected. It consisted of dilation of the veins by using compliant-type balloon catheters. Catheter venography was repeated at the end of the therapeutic procedure to document the immediate outcome of the treatment.
Of the 10 patients with MS who underwent the PTA treatment, 3 presented with $\geq 2$ venous hemodynamic criteria at the 6-month follow-up and were diagnosed with restenosis. However, we detected at least 1 venous anomaly on Doppler sonography in $50 \%$ of the treated patients. This suggests that the PTA approach for treatment of IJV anomalies has a somewhat limited value. The B-mode abnormalities (flaps, membrane, malformed valve, septa, and web) that do not cause apparent extraluminal stenoses but contribute to altered intraluminal flow are difficult to treat with compliant-type balloon catheters. All 5 patients with MS who presented with Doppler sonography abnormalities at follow-up had these types of anomalies. Use of specifically developed stents for IJVs or cutting balloon angioplasty may be a possible solution for treatment of these types of venous anomalies. The safety and efficacy data from the EVTMS study will be the subject of future reports.

A major limitation of all studies of diagnostic accuracy is the necessity to refer to a criterion standard. In our case, we are referring to catheter venography (Table 3 ). However, in this particular field, catheter venography can, on occasion, also present limitations. For instance, if malformed and/or reversed valve cusps are crossed by the catheter, the malformed valve can be kept open artificially, thereby preventing docu- 

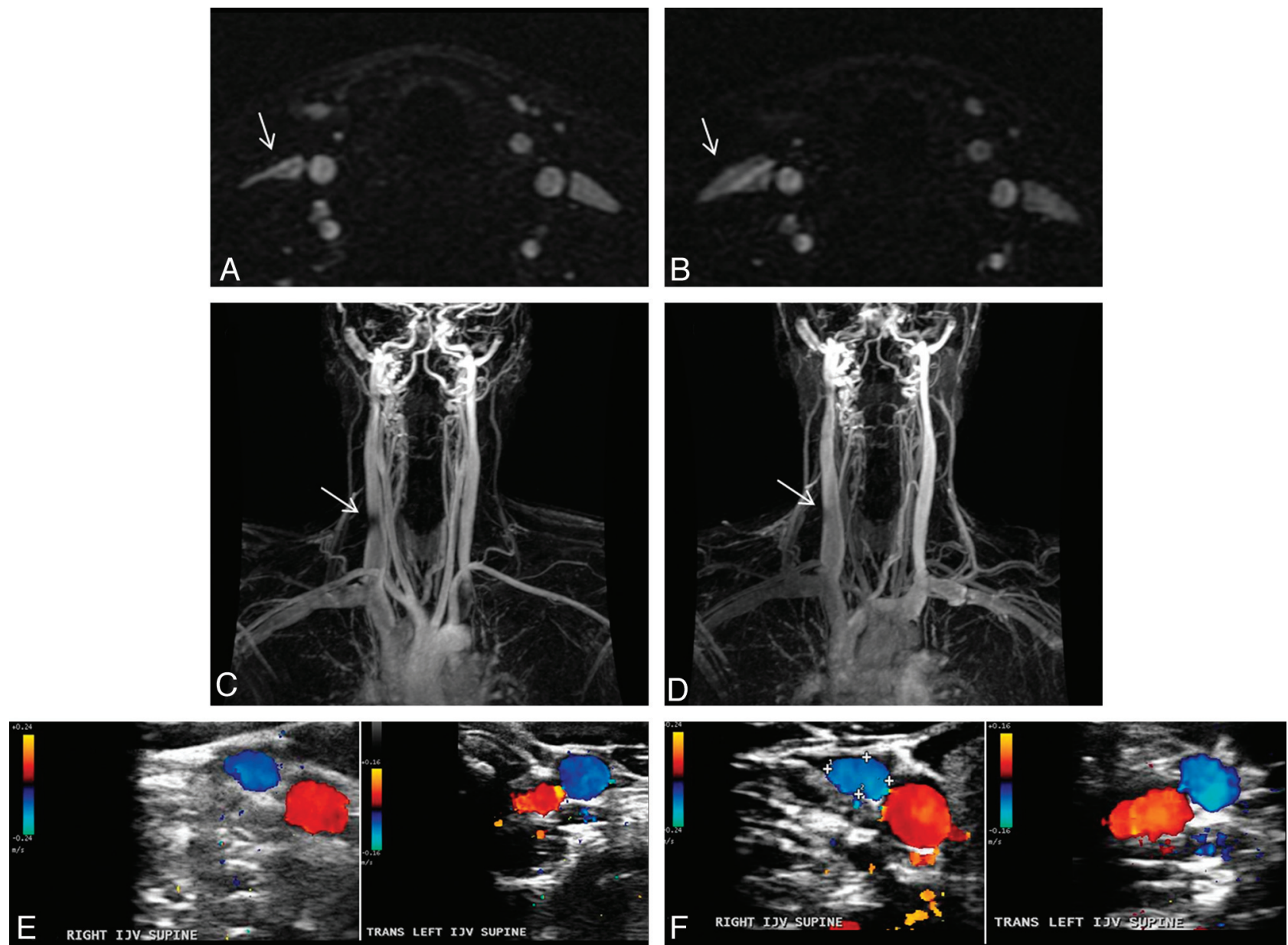

Fig 4. Variability between the baseline $(A, C$, and $E$, TOF, TRICKS, and Doppler sonography, respectively) and follow-up ( $B, D$, and $F$, TOF, TRICKS and Doppler sonography, respectively) examinations in a 39-year-old healthy male control. Flattening of the right IJV (arrows) present at the baseline $(A$ and $C$ ) examination is not present at follow-up ( $B$ and $D$ ). Doppler sonography examination shows normal findings at baseline $(E)$ and follow-up $(F)$

\begin{tabular}{|c|c|c|c|c|}
\hline $\begin{array}{l}\text { Table 3: } \\
\text { TRICKS) } \\
\text { (criterion }\end{array}$ & $\begin{array}{l}\text { ensitivity, spe } \\
\text { d Doppler so } \\
\text { tandard) for }\end{array}$ & $\begin{array}{l}\text { ificity, PPV, a } \\
\text { ography relat } \\
\text { tection of IJV }\end{array}$ & $\begin{array}{l}\text { NPV of MR } \\
\text { e to cathete } \\
\text { anomalies }\end{array}$ & $\begin{array}{l}\text { rof and } \\
\text { nography }\end{array}$ \\
\hline $\begin{array}{l}\text { Measure/ } \\
\text { Side }\end{array}$ & $\begin{array}{c}\text { Sensitivity } \\
(\%)\end{array}$ & $\begin{array}{c}\text { Specificity } \\
(\%)\end{array}$ & PPV (\%) & NPV (\%) \\
\hline$\overline{\mathrm{TOF}}$ & & & & \\
\hline Right & $99(44-100)$ & $33(3-51)$ & $45(12-65)$ & $99(21-100)$ \\
\hline Left & $99(21-100)$ & $45(12-065)$ & $33(3-51)$ & $99(44-100)$ \\
\hline Both & $99(51-100)$ & $33(10-49)$ & $33(10-49)$ & $99(51-100)$ \\
\hline TRICKS & & & & \\
\hline Right & 99 (44-100) & 44 (8-64) & $49(14-69)$ & $99(34-100)$ \\
\hline Left & $99(21-100)$ & $45(12-65)$ & $33(3-51)$ & $99(44-100)$ \\
\hline Both & $99(51-100)$ & $39(14-56)$ & $35(11-52)$ & $99(57-100)$ \\
\hline DS & & & & \\
\hline Right & $79(21-94)$ & $100(65-100)$ & $99(34-100)$ & $90(53-98)$ \\
\hline Left & $99(21-100)$ & $100(70-100)$ & $99(21-100)$ & $100(70-100)$ \\
\hline Both & $82(30-95)$ & $100(81-100)$ & $99(44-100)$ & 95 (73-99) \\
\hline
\end{tabular}

a $95 \%$ confidence intervals are shown in parentheses for all values.

mentation of stenosis. Moreover, recognition of intraluminal B-mode abnormalities requires experience and proper training.

We previously reported that the inter-rater variability rate between trained and untrained Doppler sonography operators substantially increased after training was performed. ${ }^{11}$ The interobserver agreement between trained operators was much more reliable $(\kappa=0.80)$ compared with the agreement between untrained operators $(\kappa=0.47)$. Therefore, the Doppler sonography protocol requires appropriate training before it can be applied.

The MRV techniques easily and globally depict the extracranial venous system compared with Doppler sonography and catheter venography. However, in contrast to high-resolution Doppler sonography and catheter venography, MRV techniques do not have sufficient resolution to show vessel wall or intraluminal B-mode abnormalities such as the annulus, flaps, webs, and so forth. This is one of the main limitations of comparing conventional MRV with Doppler sonography and catheter venography. We considered pinpoint and absent flow on TOF and TRICKS to be abnormal findings in IJVs. We did not consider flattening to be a pathologic finding because, in a previous study, it had the same appearance and approximate frequency in healthy controls as in patients with MS. ${ }^{6}$ Flattening was quite a common finding at the level of the lateral masses of the atlas and at the thyroid gland level

In patients with MS, TOF and TRICKS showed high sensitivity and NPV but low specificity and PPV relative to catheter venography. No agreement was found between TOF and catheter venography in $70 \%$ of patients with MS and between TRICKS and catheter venography in $60 \%$ of patients with MS. More than $50 \%$ of the healthy controls did not show agree- 
ment between MRV and Doppler sonography findings, confirming results observed in patients with MS pretreatment. Both in patients with MS and healthy controls, no agreement was found between MRV and Doppler sonography at the 6-month follow-up.

The lack of dynamism in real-time of conventional MRV is an important disadvantage when studying veins because, in contrast to arteries, veins are prone to morphologic and hemodynamic changes under multiple circumstances. MRV is performed only in the supine position, and consequently, it misses all the dynamic information provided by Doppler sonography in the upright position. For this reason, a static image of a vein in a certain moment can be misleading and it should be considered with caution when contemplating stenosis. Veins have a tendency to collapse and change their morphology and size depending on hydration status, position (gravitational variability), intrathoracic pressure (respiration, Valsalva), cardiac status, and compression from adjacent structures. ${ }^{12-15}$ In particular, a blank jugular vein with a block of the flow due to a stenosing lesion of the outlet has reduced transmural pressure. In this condition, it becomes easily compressed by adjacent structures. Consequently, when one conducts MRV, changes in the morphology of the IJVs could be attributed to several factors: different respiratory phases during sequence acquisition, different positioning of the head and neck coil, changes in the contact points (extrinsic compression) with the coil, changes due to swallowing movements, physiologic stenosis of the left brachiocephalic vein during regular breathing in the supine position, ${ }^{16-20}$ and different cerebral drainage patterns, as described in the horizontal position in healthy controls (jugular drainers versus nonjugular drainers). ${ }^{13,21}$ This may explain why we found variability in the morphology of the IJVs between TOF and TRICKS. Both examinations were conducted at the same scanning session, and this finding applied to healthy controls as well as patients with MS. MRV variability was also detected at follow-up examinations in healthy controls.

The MR imaging techniques we used are relatively standard, and other more advanced approaches are possible. These include phase-contrast MRV, ${ }^{22}$ which is capable of simultaneous flow quantification in multiple directions, and multidirectional TOF MRV, which could potentially alleviate some of the usual MRV artifacts and provide more detailed flow information. Although they still lack the positional dynamism of Doppler sonography, MRV techniques like these might provide more sensitivity and/or specificity for detection of venous anomalies. However, these techniques are complex and more technically challenging, perhaps too much so to be applied to routine clinical practice. The focus of our current investigation was to understand the value of conventional MRV. Our results speak to that and leave open the possibility that more nonconventional MRV approaches may lead to different results.

Apart from the anomalies described in the IJVs, extracranial venous collateral circulation was previously described as a compensatory mechanism of CCSVI in patients with MS. ${ }^{1,3}$ We were not able to compare collateral circulation between the 3 imaging techniques because our original protocol did not systematically evaluate asymmetries and prominence.

Although the azygous vein is another important target for the diagnosis and treatment of CCSVI, ${ }^{1,3}$ we did not analyze this venous segment in the present study. During the development of the MRV protocol, we tried to image the azygous vein, but the quality of the protocol was very low and did not reliably assess the morphology of the azygous vein. Primarily, this was due to the fact that we did not use cardiac gating and the FOVs were centered on the neck. Moreover, higher concentrations of contrast than we applied are needed for proper visualization of the azygous vein. Therefore, the diagnostic value of MRV for assessment of the azygous vein needs further technical improvement.

The differences between various imaging modalities in our study emphasize the need for a multimodal approach for assessment of CCSVI. At this time, no standardized consensus definitions are available that describe venous pathology. There is also a lack of diagnostic reference guidelines related to the evaluation of the extracranial cerebrospinal venous system and collateral circulation. Use of intraluminal Doppler meth$\mathrm{ods}^{23}$ and pathologic approaches can provide more evidence on whether CCSVI exists in MS.

\section{Conclusions}

This study suggests that conventional MRV has limited value for the detection of IJV anomalies in patients with MS. A repeat of the examination in the same individuals showed modest-to-low stability of MRV for longitudinal assessments. Therefore, its use for follow-up of patients after endovascular treatment is limited. These findings need further confirmation in larger case-control studies. They emphasize the need for further research and discussion of the findings as well as determining the criteria to be considered and the best imaging techniques to be used.

\section{Acknowledgments}

We thank all subjects participating in the study as well as the other study contributors who were involved in this project. We also thank Eve Salczynski and Kresimir Dolic for technical assistance in the preparation of this manuscript.

\section{References}

1. Zamboni P, Galeotti R, Menegatti E, et al. Chronic cerebrospinal venous insufficiency in patients with multiple sclerosis. J Neurol Neurosurg Psychiatry 2009;80:392-99

2. Zamboni P, Menegatti E, Galeotti R, et al. The value of cerebral Doppler venous haemodynamics in the assessment of multiple sclerosis. J Neurol Sci 2009;282: 21-27. Epub 2009 Jan 13

3. Zamboni P, Galeotti R, Menegatti E, et al. A prospective open-label study of endovascular treatment of chronic cerebrospinal venous insufficiency. $J$ Vasc Surg 2009;50:1348-58 el-3

4. Lee AB, Laredo J, Neville R. Embryological background of truncular venous malformation in the extracranial venous pathways as the cause of chronic cerebro spinal venous insufficiency. Int Angiol 2010;29:95-108

5. Al-Omari MH, Rousan LA. Internal jugular vein morphology and hemodynamics in patients with multiple sclerosis. Int Angiol 2010;29:115-20

6. Hojnacki D, Zamboni P, Lopez-Soriano A, et al. Use of neck magnetic resonance venography, Doppler sonography and selective venography for diagnosis of chronic cerebrospinal venous insufficiency: a pilot study in multiple sclerosis patients and healthy controls. Int Angiol 2010;29:127-39

7. Simka M, Zaniewski M. Reinterpreting the magnetic resonance signs of hemodynamic impairment in the brains of multiple sclerosis patients from the perspective of a recent discovery of outflow block in the extracranial veins. J Neurosci Res 2010;88:1841-45

8. Zamboni P, Galeotti R, Weinstock-Guttmann B., et al. Endovascular treatment for chronic cerebrospinal venous insufficiency in multiple sclerosis: a longitudinal pilot study. Mult Scler 2009;15:P483:S141

9. Lublin FD, Reingold SC. Defining the clinical course of multiple sclerosis: 
results of an international survey-National Multiple Sclerosis Society (USA) Advisory Committee on Clinical Trials of New Agents in Multiple Sclerosis. Neurology 1996;46:907-11

10. Kurtzke JF. Rating neurologic impairment in multiple sclerosis: an expanded disability status scale (EDDS). Neurology 1983;33:1444-52

11. Menegatti E, Genova V, Tessari M, et al. The reproducibility of colour Doppler in chronic cerebrospinal venous insufficiency associated with multiple sclerosis. Int Angiol 2010;29:121-26

12. Beddy $\mathrm{P}$, Geoghegan $\mathrm{T}$, Ramesh $\mathrm{N}$, et al. Valsalva and gravitational variability of the internal jugular vein and common femoral vein: ultrasound assessment. Eur J Radiol 2006;58:307-09

13. Doepp F, Schreiber SJ, von Munster T, et al. How does the blood leave the brain? A systematic ultrasound analysis of cerebral venous drainage patterns. Neuroradiology 2004;46:565-70

14. Escott EJ, Branstetter BF. It's not a cervical lymph node, it's a vein: CT and MR imaging findings in the veins of the head and neck. Radiographics 2006;26:1501-15

15. Schreiber SJ, Lurtzing F, Gotze R, et al. Extrajugular pathways of human cerebral venous blood drainage assessed by duplex ultrasound. J Appl Physiol 2003;94:1802-05

16. Kudo K, Terae S, Ishii A, et al. Physiologic change in flow velocity and direction of dural venous sinuses with respiration: MR venography and flow analysis. AJNR Am J Neuroradiol 2004;25:551-57
17. Tanaka T, Uemura K, Takahashi M, et al. Compression of the left brachiocephalic vein: cause of high signal intensity of the left sigmoid sinus and internal jugular vein on MR images. Radiology 1993;188:355-61

18. Tseng YC, Hsu HL, Lee TH, et al. Venous reflux on carotid computed tomography angiography: relationship with left-arm injection. J Comput Assist Tomogr 2007;31:360-64

19. Uchino A, Nomiyama K, Takase $Y$, et al. Retrograde flow in the dural sinuses detected by three-dimensional time-of-flight MR angiography. Neuroradiology 2007;49:211-15

20. Yakushiji Y, Nakazono T, Mitsutake S, et al. Sonographic findings of physiologic left brachiocephalic vein compression in a case initially misdiagnosed as a left internal jugular vein thrombus. J Ultrasound Med 2009;28:253-58

21. Hoffmann O, Klingebiel R, Braun JS, et al. Diagnostic pitfall: atypical cerebral venous drainage via the vertebral venous system. AJNR Am J Neuroradiol 2002;23:408-11

22. van Amerom JF, Vidarsson L, Wu S, et al. Regional pulmonary blood flow: comparison of dynamic contrast-enhanced MR perfusion and phase-contrast MR. Magn Reson Med 2009;61:1249-54

23. Machida H, Komori $\mathrm{Y}$, Ueno E, et al. Spatial factors for quantifying constant flow velocity in a small tube phantom: comparison of phase-contrast cinemagnetic resonance imaging and the intraluminal Doppler guidewire method. Jpn J Radiol 2009;27:335-41 